\title{
Data Compression using Simulated Circular Indexing Transform (SCIT)
}

\author{
Gebremichael Girmay \\ Andhra University College of Engineering (A), \\ Andhra University, Visakhapatnam-530003, India
}

\author{
D. Lalitha Bhaskari \\ Andhra University College of Engineering (A), \\ Andhra University, Visakhapatnam-530003, India
}

\begin{abstract}
One of the critical issues in big data environment is the volume of the data generated and streamed in real time or archived for later use. If the data streamed in real-time is large enough then the time required to transmit that data may lead to unnecessary delay and latency problems. The other case with huge volumes of data when it is archived is the case of storage devices requirements. Compressing the data before or while transmitting is one useful solution to minimize the size of data and thus avoid or minimize the latency problem and storage shortages. In this research paper the focus will be to deal on some compression techniques, especially in data transforming techniques similar to BWT, MTF and RLE which are commonly used to transform the data prior to encoding the data into fewer bits using entropy encoding techniques such as Huffman, Arithmetic, Golomb, etc. All the data transforming techniques have their own positive and weak sides. Thus in this paper alternative method is proposed to fill some of the gaps that cannot be solved by the already existing data compression transforming techniques. The proposed algorithm can be combined with other data compression methods to optimize the compression efficiency. The performance of this proposed algorithm is measured as compared to other related compression algorithms, and it is found that in some special cases it can perform better than others.
\end{abstract}

\section{General Terms}

Data compression, transforming, modeling, entropy encoding, lossless and lossy, statistical, dictionary, performance, big data.

\section{Keywords}

Info-table, unique symbol, codeword, SCIT, frequency, gap, critical issue.

\section{INTRODUCTION}

Data compression is the process of minimizing the size of code generated by digital coding systems to represent a specific data or information. Digital coding is the process of using binary digits or bits to represent letters, characters and other symbols in a digital format. A digital system is a device or technology that generates discrete values based on some bit combination techniques, so that each character, letter or symbol is unique and easily distinguishable from each ones. There are several types of digital coding techniques widely used, which include ASCII code, BCD/EBCDIC,
UNICODE, etc. For example, using ASCII code, a digital system can generate 128 unique symbols, while using EBCDIC it can provide 256 unique symbols.

In this paper, the necessity and advantage of compression is discussed based on data transfer and data storage issues, and some well-known compression algorithms and techniques are reviewed in brief.

As stated in [1], data compression is the process of converting an input data stream (the source stream or the original raw data) into another data stream (the output, or the compressed, stream) that has a smaller size.

One of the necessities for choosing to compress data is to minimize transfer delay/latency during data communication using any media. Specifically, if the input data is generated in real time and is streamed on the way or is stored in memory and is to be transferred to any other destination at any time using speed-constrained systems.

The second issue is when there is small storage volume within the system, but the data or document is to be archived for later use. Other issues are such as to speed up the data processing operations, to reduce energy required to process or to transmit, and for security purposes as well.

Data compression works if the original file of data is re-encoded as follows:

- Reduce the number of symbols in the data using predictive methods.

- Rearrange the symbols in the data using transform techniques.

- Encode more frequent symbols with fewer bits.

RLE is an example of compression technique that works well on documents that exhibit to contain repetitive symbols. BWT, MTF, PPM, and Differencing are some examples of predictive/transform techniques used to rearrange characters or symbols within a document after which it is possible to more reduce the size of the document. Huffman coding, Arithmetic coding, Shannon-Fano, Tunstall, Elias gamma, and other more methods are those used to encode frequent symbols with fewer bits.

A particular compression method can either be lossy or lossless. With Lossless method the decompressor's output is an exact copy of the original data. Text compression is generally of lossless type. With a lossy method, the decoder's output is not an exact copy of 
the original data but an approximation that is somewhat similar to the original version. Lossy compression is useful when compressing pictures (e.g., JPEG) or audio files (e.g., MP3), where small deviations from the original are invisible (or inaudible) to the human senses [2].

Codes can be categorized as block-block, block-variable, variableblock, or variable-variable [3]. Compression coding techniques such as Huffman and Arithmetic generate variable-length codeword for every unique symbol or group of symbols. Coding methods such as ASCII generate fixed-length codes for every unique character.

In this paper we focus exclusively on lossless compression algorithms, by modeling and implementing a new transform technique similar to MTF method and encoding using Huffman coding as a final step.

Most compression/decompression systems apply a combination of several techniques to achieve better compression efficiency. Compression/decompression performance is measured based on the data type compressed, and whether it is lossy or lossless compression. Commonly used performance measurement methodologies include: compression ratio, compression factor, compression gain, compression rate, and fidelity. In most cases, if possible, the goal is to achieve a best compression factor and speed with less degrading fidelity; Entropy being a reference for better encoding factor, as defined by Shannon.

In subsequent sections and subsections, the basic concepts of compression will be covered in some detail. Section 2, literature review, Section 3, modeling and coding and Section 4, proposed technique and its implementation will be covered.

\section{LTITERATURE REVIEW}

The research works done in the compression/decompression area is wide, and is still continuing in several fields as the data generated is vastly growing at high rate. In this chapter some basic theoretical and practical concepts and approaches are reviewed. Generally compression can be lossless or lossy. Lossless compression is concerned with textual documents and some critical data such as medical scanning images. Lossless data compression methods can be classified as Entropy type, Dictionary type, and other types [1].

In entropy (or statistical) coding each symbol is assigned a code based on the probability model that is chosen. Highly probable symbols are assigned short codes, and vice versa. In dictionary coding, groups of consecutive characters, words, or phrases are replaced with seemingly short code. The group of symbols or words represented by the corresponding codes can be found by referring it in the dictionary.

Lossy data compression goes with general images, videos and audios. Next some commonly used optimal encoding algorithms are detailed, tuning towards the proposed method. Indeed saving storage space is a common reason for the use of compression [4], and other reasons are of course speed and band width utilization.

\subsection{Huffman Coding}

Huffman coding, developed, in 1952, by D. Huffman, is a popular method for data compression, categorized under the entropy coders. An entropy coder is a method that assigns to every symbol from the alphabet a code depending on the probability of symbol occurrence [5]. The symbols that are more probable to occur get shorter codes than the less probable ones. Huffman coding is an optimal coding technique for compression purpose and serves as the basis for several popular programs run on various platforms [6]. Some programs use just the Huffman method, while others use it as one step in a multistep compression process. The Huffman method is somewhat similar to the Shannon-Fano method. Huffman coding is one of the best methods for coding with respect to probabilistic model.

Though the size of the code assigned to a symbol $a_{i}$ basically depends on its probability of occurrences $p_{i}$ it is also affected by the number of unique symbols (the size of the alphabet) in the stream or document. A small alphabet requires just a few codes, so they can all be short; a large alphabet requires many codes, so some must be long. One critical issue in Huffman coding is that, if those unique symbols (whether they are few or more) that constitute a given data file, do appear with equal probabilities (or frequency of occurrence), it may not considerably change the size of the document. For example, if there are $n$ unique symbols and $n=2^{m}$, where $m$ is a common code length (e.g. for ASCII code, $m=7, n=128$ ) supported by the system when generating each alphanumeric characters and symbols, applying Huffman coding will not affect the original size of the document. Thus in such related cases some transform or predictive techniques are required either to:

- reduce the number of unique symbols, or

- vary the frequency of the representative symbols, or

- rearrange their position in the document so that produce a repetitive form.

What it means is, the Huffman method cannot assign to any symbol a code shorter than one bit, if some form of predictive transform is not done ahead to using Huffman coding. Therefore to achieve meaningful compression process, a need comes to apply a multistep compression process, the last step being Huffman or other statistical coding methods such us Arithmetic, Elias, Golomb, and more others. The multistep compression process may incorporate RLE, BWT, MTF, and PPM, etc. compression techniques that can be applied prior to conducting Huffman or the other statistical coding methods.

Huffman Decoding: During compressing some basic information must be collected in the form of Information Table (Info-table). These points (info) include: list of the unique symbols and their probability (or frequency); and if required, their variable-size codeword, the size of the original and compressed file. During decoding the compressor (encoder) has to determine the codes based on the supplied points in the info-table. Basically it does that based on the probabilities (or frequencies of occurrence) of the symbols. The Huffman decompressor (decoder) must first construct (map) the Huffman tree as was constructed by the compressor (encoder). Only then can it read and decode the compressed stream. Then, if a multistep compression was used, the decompressor should follow the same step but in reverse order, to gate the original stream.

\subsection{Run Length Encoding (RLE)}

RLE is one of the encoding techniques used to encode and as well to transform a data under compression. It works well on data sets with long runs of similar symbols. It can work in combination with other encoding techniques as well. RLE is used for any type of data format if such data type exhibits long runs of the specific elements in several positions, where sequences of consecutive identical elements are replaced by the length of the run [4]. The symbols in the stream may or may not be with equal probability, what is required is, there should be less randomness of the symbols. 
It replaces a run of symbols with a tuple that contains the symbol and the number of times it is repeated [7]. In general, a string $s_{1} s_{1} \ldots s_{1} s_{2} s_{2} \ldots s_{2} s_{3} s_{3} \ldots$, in which each symbol appears in a long run (as countable infinite repetitive) is suitable to reduce the runs and thus reducing(compressing) the size of the string. Example, the string "ssssiiiiooonn" can be encoded or transformed using RLE as $4 \mathrm{~s} 4 \mathrm{i} 3 \mathrm{o} 2 \mathrm{n}$. Then it can further be coded using statistical models such as Huffman or Arithmetic encoders.

One of the critical issues in using RLE is when the similarity run of symbols is short (e.g. one or two), which will result in increasing the size rather than compressing it. Anyway, RLE can be used at the beginning or after some techniques are processed in most multistep compression process, e.g., RLE-BWT-MTF-RLE-Huffman [8].

\subsection{Move-to-Front Transform}

Move-to-Front (MTF) is an adaptive scheme and works on the principle that the appearance of a symbol in the input data makes that symbol more likely to appear in the near future. As detailed in [9] the basic idea of this method is to maintain the alphabet $A$ of symbols as a list where frequently-occurring symbols are located near the front. A symbol $s$ is encoded as the number of symbols that precede it in this list. Thus if alphabet $A=(C, o, m, p, r, e, s, \ldots)$ and the next symbol in the input stream to be encoded is the $p$, it will be encoded as 3 , since it is preceded by three symbols. There are several possible variants to this method; the most basic of them adds one more step: After symbol $s$ is encoded, it is moved to the front of list $A$. Thus, after encoding the $p$, the alphabet is modified to $A=(p, C, o, m, r, e, s, \ldots)$. This move-to-front step reflects the expectation that once $p$ has been read from the input stream, it will be read many more times and will, at least for a while, be a common symbol. The move-to-front method is locally adaptive, since it adapts itself to the frequencies of symbols in local areas of the input stream. The main idea is to move to front the symbols that mostly occur, so those symbols will have smaller output number or results in to more repetitiveness and thus will be coded with short codeword bits. Example, as the input document is processed, each symbol (or word, when used at world level) is looked up in the alphabet list and if it happens to occur as the $i^{t h}$ entry, it is coded by the index number $i$. Then the symbol is moved to the front of the list so that if it occurs soon afterwards, it will be coded by a number smaller than $i$.

This technique is intended to be used in combination with other compression techniques like Burrows-wheeler transform (BWT).

After a stream is transformed using MTF, then follows encoding it using either of the variable length entropy encoders such as Golomb, Huffman, arithmetic, etc.

An optional example is the number $i$ is coded so that the smaller its value the shorter its code, and thus more likely symbols are represented more compactly.For instance, one suitable method of assigning variable code lengths is to code position index integer number $i \geq 1$ as the binary representation of $i$ prefixed by $\left\lfloor\log _{2}^{i}\right\rfloor$ zeros. In this case as the binary code of the integer $i$ is $1+\left\lfloor\log _{2}^{i}\right\rfloor$ bits long, then adding $\left\lfloor\log _{2}^{i}\right\rfloor$ zeros becomes $1+2\left\lfloor\log _{2}^{i}\right\rfloor$ bits [1]. For example the codes for $i$ is $1,2,3$, and 4 are 1, 010,011, and 00100 , respectively.

Therefore this method produces good results if the input stream contains concentrations of identical symbols, i.e. if the stream satisfies the concentration property (if the local frequency of symbols changes significantly from area to area in the input stream).
It can be shown that the move-to-front method performs, in the worst case, slightly worse than Huffman coding. At best, it performs significantly better when it is used with BWT or when the frequencies of the symbols in the input file are or not varied meaningfully, but appearing repetitively.

There are several possible variants to this method:-

Move-ahead- $k$ - The element of $A$ matched by the current symbol is moved ahead $k$ positions instead of all the way to the front of $A$.

Wait-c-and-move- An element of $A$ is moved to the front only after it has been matched $c$ times to symbols from the input stream (not necessarily $c$ consecutive times).

A critical issue in using MTF is, it gives poor performance if request sequence is in reverse order of the alphabet list [10]. That is if the distribution of the symbols in the stream appears in reverse order at seemingly equal interval, then using MTF will not achieve good compression performance.

\subsection{Burrows-Wheeler-Transform (BWT)}

As explained in $[9,11]$, BWT method was developed by Michael Burrows and David Wheeler in 1994, while working at DEC Systems Research Centre in Palo Alto, California. The main idea of the BWT method is that it starts with a string $S$ of $m$ symbols and scramble (permute) them into another string $L$ that satisfies two conditions.

(1) Any region of $L$ will tend to have a concentration of just a few symbols. Another way of saying this is if a symbol $s$ is found at a certain position in $L$, then other occurrences of $s$ are likely to be found nearby. This property means that $L$ can easily and efficiently be compressed with the move-to-front method, perhaps in combination with RLE and then apply a statistical model(e.g. Huffman or others) to assign probabilities to the transformed symbols.

(2) It is possible to reconstruct the original string $S$ from $L$ (a little more data may be needed for the reconstruction, in addition to $L$, but not much).

One of the critical issues is that, the BWT method will work well only if $m$ is large (at least several thousand symbols per string) [9]. Another case is that, as a string with $m$ symbols can have $m$ ! permutations, it becomes a large number for large value of $m$, thus the particular permutation used by BWT has to be carefully selected.

The BW codec proceeds in the following steps:

(1) String $L$ is created, by the encoder, as a permutation of $S$. Some more information, denoted by $I$, is also created, to be used later by the decoder in step 3 .

(2) The encoder compresses $L$ and $I$ and writes the results on the output stream. This step typically starts with RLE, continues with move-to-front coding, and finally applies Huffman coding.

(3) The decoder reads the output stream and decodes it by applying the same methods as in 2 above but in reverse order. The result is string $L$ and variable $I$.

(4) Both $L$ and $I$ are used by the decoder to reconstruct the original string $S$.

Given an input string of $m$ symbols, the encoder constructs an $m \times$ $m$ matrix where it stores string $S$ in the top row, followed by $(m-$ 1 ) copies of $S$, each cyclically shifted (rotated) one symbol to the left. The matrix is then sorted lexicographically by rows. 
The permutation $L$ selected by the encoder is the last column of the sorted matrix. It should be noted that the Burrows-Wheeler method can easily achieve efficient compression when applied to longer strings (thousands of symbols), though it requires more memory and time to do that. Thus WBT works in block wise.

\subsection{Entropy}

Entropy is one of the important concepts in information theory. It is a theoretical measure of quantity of information $[5,6,7,9,12]$. Entropy is a measure of the amount of order or redundancy in a message. The value of entropy is small if there is a lot of order and otherwise it is large if there is a lot of disorder. Entropy as a measure is very important with regard to achieving better data compression goals, because the length of a message after it is compressed (encoded) should ideally be equal to its entropy. That is entropy gives an estimate of how long to make the encoded version of each symbol in a massage.

As Shannon demonstrated, for a set of possible events with known probabilities $p_{1}, p_{2}, p_{3}, \ldots, p_{n}$, that sum to 1 , the Entropy of these events is given as

$$
E\left(p_{1}, p_{2}, p_{3}, \ldots, p_{n}\right)=-k \sum_{i=1}^{n} p_{i} \log _{2}^{p_{i}}
$$

where, the positive constant $k$ governs the units in which entropy is measured. As normally, the units are bits, where $k=1$, and logs are taken with base 2 , then:

$$
E=-\sum_{i=1}^{n} p_{i} \log _{2}^{p_{i}}
$$

The information content or entropy of a selected probability $p_{i}$ is thus given as

$$
E_{i}=-p_{i} \log _{2}^{p_{i}}
$$

From this it is clear that more likely symbols or messages having greater probabilities contain less information.

\section{MODELING AND CODING}

The task of finding a suitable model for text compression is an extremely important problem. As shown in Figure 1, some basic steps are conducted to reach the final compression step. The input message can be transformed first ahead to applying prediction. The separation into modeller and encoder is valuable because modeling and coding are very different sorts of activity [4]. The modeller determines the probability of the unique symbols in the text and supplies to the encoder. Once prediction is applied, the encoder receives the predicted probability or frequency of occurrences together with the actual input stream and turns them into sequence of bits (binary digits) to be transmitted or saved.

There are three ways that the encoder and the decoder can maintain the same model: static, semi-adaptive, adaptive modeling.

In general in statistical compression process the stream can be first transformed so by doing that the size of the stream and/or the number of unique symbols can be minimized or their frequency can be more skewed as is observed in RLE, BWT, MTF, etc. This paper work is also focusing on the transforming part of the statistical encoding.

Text Compression:

As mentioned in the previous sections some context-based text

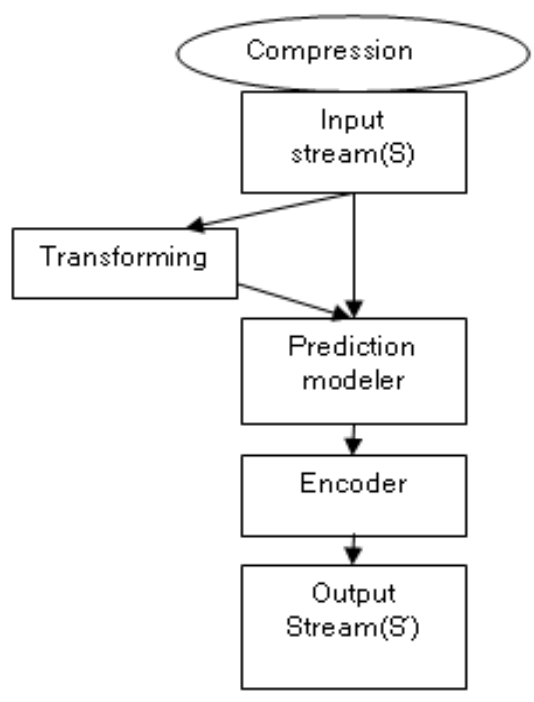

Fig. 1. Outline of Basic Steps in Lossless Compression

compression methods perform a transformation on the input data and then apply a statistical model to assign probabilities to the transformed symbols. Good examples of such methods are the Burrows-Wheeler method, also known as the Burrows-Wheeler transform.

Most text compression methods are either statistical or dictionary based $[9,13]$. In dictionary based method the text is fragmented into words and is saved in a data structure called a dictionary. When a fragment of new text is found to be identical to one of the dictionary entries, a pointer or a symbol that identify to that entry is written on the compressed stream, to become the compression of the new fragment. In statistical method, on the other hand, consists of methods that develop statistical models of the text as discussed above. The model can be static or dynamic (adaptive). A static model uses fixed probabilities, whereas a dynamic model modifies the probabilities on the fly while text is being input and compressed. Most models are based on one of two approaches: Frequency and Context. In context-based model, the modeller considers the context of a symbol when assigning it a probability. Since the decoder does not have access to future text, both encoder and decoder must limit the context to past text, i.e., to symbols that have already been input and processed.

\section{PROPOSED METHOD: SIMULATED CIRCULAR INDEXING TRANSFORM (SCIT)}

If the probabilities of the symbols in a document are more skewed, then applying statistical coding methods directly can achieve good results. The fact that the probability is skewed implies low entropy which in turn implies the possibility of very good compression.

The proposed method, here and onward called, Simulated Circular Indexing Transform (SCIT), aims at filling some specific gab observed in the compression or data transforming techniques reviewed above. The approach is similar to the Move-to-Front transforming techniques. But instead of moving the symbol to any location in the list of alphabets, is just to play with index, moving or stepping next or back circularly if a different symbol is coming otherwise don't move if the current symbol happens to occur 
repetitively. Conceptually all the moves (next/back or up/down) are mapped or simulated circularly to the position index value of the symbols in the alphabet, except that don't move or 'stay there' case is given a 0 index value for a repetitive symbol.

In move-to-front and some other transforming methods, the transformed data is represented by the indexes (numerical values). In the proposed SCIT method, the index is translated to the symbol currently indexed, thus the transformed data is represented by the symbol themselves. As this translated (virtual) indexing works right, for comparison and compatibility purpose, the MTF is also implemented using this concept in this work. For example take the input string is:

S: Compression is very important.

Here, $S=30$ characters, and the unique symbols or alphabets are $n=15$ as seen in the info-table: Info-table basically contains

$P 0, I a \rightarrow$

\begin{tabular}{|l|l|l|l|l|l|l|l|l|l|l|l|l|l|l|}
\hline 0 & 1 & 2 & 3 & 4 & 5 & 6 & 7 & 8 & 9 & $\mathrm{a}$ & $\mathrm{b}$ & $\mathrm{c}$ & $\mathrm{d}$ & $\mathrm{e}$ \\
$\mathrm{C}$ & $\mathrm{o}$ & $\mathrm{m}$ & $\mathrm{p}$ & $\mathrm{r}$ & $\mathrm{e}$ & $\mathrm{s}$ & $\mathrm{i}$ & $\mathrm{n}$ & $\uparrow$ & $\mathrm{v}$ & $\mathrm{y}$ & $\mathrm{t}$ & $\mathrm{a}$ &. \\
\hline
\end{tabular}

unique symbols in S and may include their codeword and some additional information, such as frequencies of each unique symbol. Let use these variables: $n, I a, P v, P 0$; where

$n$-total number of unique symbols in the stream $S$.

$I a$-holds the index value of a next symbol to be accessed, in the infotable.

$P v$-virtual(simulated) index, in terms of numeric or the symbols themselves.

$P 0$-actual index value of immediate previously accessed symbol.

Now, we can formulate the proposed method as follows. Initially $I a, P v$, and $P 0$ are assigned to a specific value, example 0 index values.

a) During encoding: For each byte in the stream find corresponding $P v$ as follows.

$P v=I a-P 0$, if $I a \geq P 0$; otherwise, $P v=(n+I a)-P 0$, if $I a<P 0$.

$$
P 0=(P v+P 0) \bmod (n)
$$

Example

Ia $\rightarrow$

S: Compression $\uparrow$ is $\uparrow$ very $\uparrow$ i m portant.=30(15)

Pv: $011111101971 \mathrm{de} 31$ a e $7 \mathrm{dda} 1 \mathrm{~d} 381$ a $42=30(11)$

P0: 012345667189769 a 54 b9 $72314 \mathrm{~cd} 8 \mathrm{ce}$

Or, $P v$ is represented by the name of the symbols themselves instead of using numeric values (Note: Here, vertical arrow $\uparrow$ replaces space),

Pv: Co o o o o $\mathrm{Co} \uparrow$ i o a.pov. i a vo a p nov r m $=30(11)$

Representing $P v$ in this way avoids writing two or more digit index values. The successive list of $P v$ gives the transformed variant of that $S$. The value 0 in the $P v$ list is to mean don't move, that means take the current symbol, rather. Since, a pointer at starting time, in most cases, points to the first byte/word in an input stream or document, the first 0 in $P v$ tells to consider the first character in $S$ which in this illustration is, $\mathrm{C}$ character.

Now as a last step is to code $P v$ list (transformed stream, $S^{\triangleleft}$ ) using either Huffman or other statistical coding techniques. This encoded or compressed document as well including the info-table is then to be communicated or archived as was intended.

Note that the number inside the parenthesis, e.g., 15 in 30(15), 11 in 30(11) represents the number of unique symbols in the original and transformed strings respectively.

b) During decoding: Huffman or other coded doc is decoded or decompressed to obtain $P v$ list back. Then retransform $P v$ list to get back $S$, as follows.

First translate each $P v$ element or symbol to corresponding numeric value one at a time.

Next determine the corresponding actual index $I a$ or $P 0$ and refer the symbol at that location in the info-table and form $S$ in sequence character by character.

$$
P 0=(P v+P 0) \bmod (n), I a \leftarrow P 0
$$

Example,

Pv: Co o o o o Co $\uparrow$ ioa.pov. i a vo a p novrm $=30(11)$

Pv: $0 \begin{array}{llllllll} & 1 & 1 & \ldots\end{array}$

P0: $012 \ldots$

Ia $\rightarrow$

S: Compression $\uparrow$ is $\uparrow$ very $\uparrow$ i m portan t . $=30(15)$

For the above transforming and retransforming example, Move-toFront, will do as follows.

Ia $\rightarrow$

S: Compression $\uparrow$ is $\uparrow$ very $\uparrow$ important $=30(15)$

Pv: 012345607689342 a 67 b 46 a a $6 \mathrm{cdc} 2 \mathrm{e}=30(15)$ i.e., using the symbols instead,

Pv: Co m pres Cien $\uparrow$ prmvsiyrs v v v s tat m. =30(15)

We can see that the number of unique symbols after transforming using the SCIT method is 11 while after using MTF is 15 same as in the original document.

As is observed in the info-table, Table 1, the transformed string may have less number of unique symbols (Syb.) as compared to the original string. This phenomenon holds true also for MTF in other examples. And also some of the symbols will gain highest frequency (Freq.) though in some cases, the SCIT technique propagates and equalizes the frequency for many symbols in which case the performance will decrease. Now when we apply the statistical encoding technique, Huffman coding, then,

$\mathbf{S} \rightarrow 115$ bits $\approx 15$ bytes/30; if $\operatorname{Huffman}(\mathrm{HM})$ is solely used.

MTF: $S^{\prime} \rightarrow 111$ bits $\approx 14$ bytes/30; if $\mathrm{MTF} \rightarrow$ HM multistep is used. SCIT: $S^{\prime} \rightarrow 91$ bits $\approx 12$ bytes/30; if SCIT $\rightarrow$ HM multistep is used.

\section{Observations:}

(1) Using Huffman without using SCIT or MTF methods perform optimally in most normal texts or cases.

(2) Using MTF in combination with BWT performs better than Huffman in many cases. i.e., BWT $\rightarrow$ MTF $\rightarrow$ Huffman

(3) Using SCIT method with or without BWT performs better than Huffman and MTF for special cases only. SCIT $\rightarrow$ Huffman or $\mathrm{BWT} \rightarrow$ SCIT $\rightarrow$ Huffman

Let examine more examples that are special cases:

S1: aabbbccddeefffgghh $=18(8)$ bytes

Huffman: $\rightarrow 54$ bits $\approx 7$ bytes/ 18

MTF: aabaacadaeafaagaha $=18(8)$ bytes $\rightarrow 38$ bits $\approx 5$ bytes $/ 18$

SCIT: aabaababababaababa $=18(2)$ bytes $\rightarrow 18$ bits $\approx 3$ bytes $/ 18$ 
Table 1. Combined Info-Table of Input, SCIT and MTF

\begin{tabular}{|c|c|c|c|c|c|c|}
\hline \multirow{2}{*}{$\begin{array}{c}\text { Po, } \\
\text { Ia }\end{array}$} & \multicolumn{2}{|c|}{ Input } & \multicolumn{2}{c|}{ SCIT } & \multicolumn{2}{c|}{ MTF } \\
\cline { 2 - 7 } & Syb. & Freq. & Syb. & Freq. & Syb. & Freq. \\
\hline 0 & C & 1 & C & 2 & C & 2 \\
\hline 1 & o & $\mathbf{3}$ & o & $\mathbf{1 1}$ & o & 1 \\
\hline 2 & $\mathrm{~m}$ & 2 & $\mathrm{~m}$ & 1 & $\mathrm{~m}$ & 3 \\
\hline 3 & $\mathrm{p}$ & 2 & $\mathrm{p}$ & 2 & $\mathrm{p}$ & 2 \\
\hline 4 & $\mathrm{r}$ & 3 & $\mathrm{r}$ & 1 & $\mathrm{r}$ & 3 \\
\hline 5 & $\mathrm{e}$ & 2 & & & $\mathrm{e}$ & 2 \\
\hline 6 & $\mathrm{~s}$ & 3 & & & $\mathrm{~s}$ & $\mathbf{4}$ \\
\hline 7 & $\mathrm{i}$ & 3 & $\mathrm{i}$ & 2 & $\mathrm{i}$ & 2 \\
\hline 8 & $\mathrm{n}$ & 2 & $\mathrm{n}$ & 1 & $\mathrm{n}$ & 1 \\
\hline 9 & $\uparrow$ & 3 & $\uparrow$ & 1 & $\uparrow$ & 1 \\
\hline $\mathrm{a}$ & $\mathrm{v}$ & 1 & $\mathrm{v}$ & 3 & $\mathrm{v}$ & 4 \\
\hline $\mathrm{b}$ & $\mathrm{y}$ & 1 & & & $\mathrm{y}$ & 1 \\
\hline $\mathrm{c}$ & $\mathrm{t}$ & 2 & & & $\mathrm{t}$ & 2 \\
\hline $\mathrm{d}$ & $\mathrm{a}$ & 1 & $\mathrm{a}$ & 4 & $\mathrm{a}$ & 1 \\
\hline $\mathrm{e}$ & $\cdot$ & 1 & $\cdot$ & 2 &. & 1 \\
\hline & \multicolumn{2}{|c|}{$\mathrm{n}=15, \mathrm{~S}=30$} & $\mathrm{n}=11, \mathrm{~S}^{\circ}=30$ & $\mathrm{n}=15, \mathrm{~S}^{\circ}=30$ \\
\hline
\end{tabular}

S2: abcdefghijklmnopqrstuvwxyzzyxwvutsrqponmlkjihgfedcba $=52$ (26) bytes

Huffman: $\rightarrow 248$ bits $\approx 31$ bytes $/ 52$

MTF: abcdefghijklmnopqrstuvwxyzabcdefghijklmnopqrstuvwxyz $=52(26)$ bytes $\rightarrow 248$ bits $\approx 31$ bytes $/ 52$

SCIT: abbbbbbbbbbbbbbbbbbbbbbbbbazzzzzzzzzzzzzzzzzzzzzzz zzz $=52(3)$ bytes $\rightarrow 79$ bits $\approx 10$ bytes $/ 52$

S3: abcdefghijklmnopqrstuvwxyzabcdefghijklmnopqrstuvwxyz $=52(26)$ bytes

Huffman: $\rightarrow 248$ bits 31 bytes/52

MTF: abcdefghijklmnopqrstuvwxyzzzzzzzzzzzzzzzzzzzzzzzzzzzz $=52$ (26) bytes $\rightarrow 170$ bits $\approx 22$ bytes $/ 52$

SCIT: abbbbbbbbbbbbbbbbbbbbbbbbbbbbbbbbbbbbbbbbbbbbbbb bbbb $=52(2)$ bytes $\rightarrow 52$ bits $\approx 7$ bytes $/ 52$

(Now if we apply more methods such as RLE just after applying SCIT, the compressed document may come down to less than 5 bytes only.)

In this last example, S3, though unique symbols in MTF seem more as compared to SCIT, the difference in the compressed outcome is just 15 bytes even though $\mathrm{S} 3$ can repeat million times in that similarity and repetitive sense of sequence. But in the first and second examples SCIT performs best as compared with Huffman alone or MTF.

\section{RESULTS \& PERFORMANCE COMPARISON}

The amount of compression that can be obtained using current techniques is in most cases a trade-off against speed and the amount of memory required. Here to evaluate the performance of the proposed transforming technique, SCIT, it is compared with MTF with and without using BWT. Especially a focus is given for the cases that the proposed technique can achieve better performance.

Commonly used benchmark test files can be found from the Calgary and Canterbury Corpus which includes text, image, and object files that can be used to evaluate lossless compression methods. Thus using some of this files and additional files it is to be demonstrated that the SCIT techniques will work better for some special arrangement or distribution of symbols in particular files.
Some of the basic points assumed to measure compression are memory required to implement the method, how speedy the method performs on a given system, the amount of compression gained, how closely the reconstruction resembles the original stream (fidelity), and the relative complexity of the algorithm. Out of these measuring factors memory and time efficiency are the main to be considered $[14,15]$.

$$
\begin{aligned}
& \text { - Compression-ratio, } \mathrm{CR}=\frac{\text { Size of the compressed stream }}{\text { Size of the input stream }} \\
& \text { - Compression-factor, } \mathrm{CF}=\frac{1}{x}=\frac{\text { Size of the input stream }}{\text { size of the compressed stream }} \\
& \text { - Compression-gain, } \mathrm{CG}=\frac{\text { Compressed file size }}{\text { Original file size }} \times 100 \% \\
& \text { - Storage } \quad \mathrm{SP}=100 \mathrm{x}(1-\mathrm{CR}) \\
& =\frac{\text { Original file size- compressed file size }}{\text { Original file size }} \times 100 \%
\end{aligned}
$$

- Compression time, $\mathrm{Tcd}=$ Total time required to process, compress and/or decompress a given stream.

- Entropy, H, and average codeword length (Acl).

For this multistep compression process, $\mathrm{C}$ program has been implemented to experiment the overall compression results.

The following tables and charts summarize the compression performance of the SCIT compression technique comparing with respect to other techniques particularly with using Huffman alone, with MTF, and in a multistep compression technique using with BWT. Here as pointed out above speed and memory storage are considered as efficiency measurement and of course it is lossless compression though it works for lossy compression as well.

From Table 2., we can see that, for most normal text documents, if we directly use Huffman without using any transforming technique performs better than MTF and the SCIT techniques, and of course MTF is the next better than the SCIT. But for some special alphanumeric character combinations the SCIT and MTF respectively performs better. Example the file "alpha.txt" is a kind of artificial file and file "alpha*.txt" is the same as file "alpha.txt" but in reverse order. Therefore the SCIT method performs best for files that are composed in reversed manner at several intervals.

Figures 2, 3, 4, and 5, demonstrate some of the performance measurement parameters from Table 2 . The tables and figures show the results of compression using Huffman (HM), MTF and SCIT techniques. Again they provided the performance comparison among these three methods (HM, MTF, and SCIT). When we look at Figure 2, the values along the vertical-axis represent the SP values which illustrates above $80 \%$ storage saving can be achieved in general. Similarly it is clearly reflected that in Figure 3, compression ratio (those across vertical-axis) for SCIT is poor in most normal texts, but best for some special correlations of symbols. Figure 4, is normally the reverse of Figure 3, and shows the number of bits or characters (in the vertical-axis) replaced by a single bit or character. Figure 5, illustrates the time (across vertical-axis, in seconds) required to process, compress and decompress a given file size in bytes. One can easily identify which method is performing better for which file and in what condition.

Note: in most the figures the file name is written instead of the file size, i.e., each file size can be referred from the corresponding table, otherwise can be replaced with real file size in bytes or Kbytes.

Next as can be observed from Table 3, when we use BWT and then Huffman will not make any change to the performance obtained from using Huffman alone. But if we use BWT $\rightarrow$ MTF $\rightarrow$ Huffman or BWT $\rightarrow$ SCIT $\rightarrow$ Huffman, for most normal text files (example English text books) MTF performs better than SCIT and Huffman 
Table 2. Compression result using $\mathrm{HM}$ only, $\mathrm{MTF} \rightarrow \mathrm{HM}$ or SCIT $\rightarrow \mathrm{HM}$

\begin{tabular}{|c|c|c|c|c|c|c|c|c|c|c|c|c|c|}
\hline \multirow{2}{*}{$\begin{array}{l}\text { Input } \\
\text { File }\end{array}$} & \multirow{2}{*}{$\begin{array}{c}\text { Input File } \\
\text { Size(Bytes) }\end{array}$} & \multicolumn{4}{|c|}{ Huffman } & \multicolumn{4}{|c|}{ MTF } & \multicolumn{4}{|c|}{ SCIT } \\
\hline & & Output & $\mathrm{CR}$ & SP & Tcd & Ouput & $\mathrm{CR}$ & SP & Tcd & Output & $\mathrm{CR}$ & SP & Tcd \\
\hline lice29 & 148,480 & 84,545 & 0.569 & 43.06 & 0.19 & 93,266 & 0.628 & 37.19 & 0.23 & 108,532 & 0.730 & 26.90 & 0.23 \\
\hline lcet10 & 419,235 & 243,876 & 0.582 & 41.83 & 0.33 & 262,804 & 0.629 & 37.31 & 0.42 & 310,208 & 0.740 & 26.01 & 0.45 \\
\hline plrabn 12 & 471,159 & 266,178 & 0.565 & 43.51 & 0.34 & 291,575 & 0.619 & 38.12 & 0.47 & 340,703 & 0.723 & 27.69 & 0.50 \\
\hline syoulik & 125,179 & 75,806 & 0.606 & 39.44 & 0.19 & 82,448 & 0.659 & 34.14 & 0.22 & 91,434 & 0.730 & 26.96 & 0.22 \\
\hline pi & $1,000,000$ & 424,883 & 0.425 & 57.51 & 0.34 & 424,886 & 0.425 & 57.51 & 0.47 & 424,812 & 0.425 & 57.52 & 0.42 \\
\hline random & 100,000 & 75,000 & 0.750 & 25.00 & 0.16 & 75,000 & 0.750 & 25.00 & 0.23 & 75,000 & 0.750 & 25.00 & 0.19 \\
\hline alpha & 100,000 & 59,615 & 0.596 & 40.39 & 0.14 & 12,515 & 0.125 & 87.49 & 0.17 & 12,500 & 0.125 & 87.50 & 0.14 \\
\hline alpha* & 100,000 & 59,615 & 0.596 & 40.39 & 0.16 & 59,613 & 0.596 & 40.39 & 0.16 & 18,988 & 0.189 & 81.01 & 0.14 \\
\hline
\end{tabular}

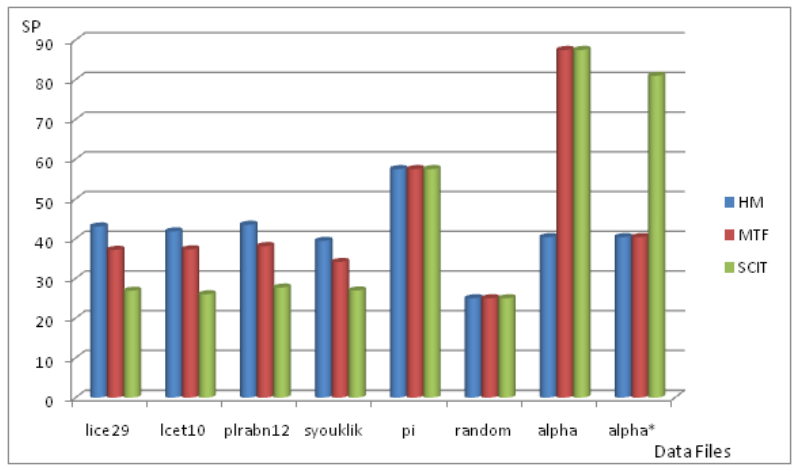

Fig. 2. Performance Comparisons based on Storage Saving Percentage (SP).

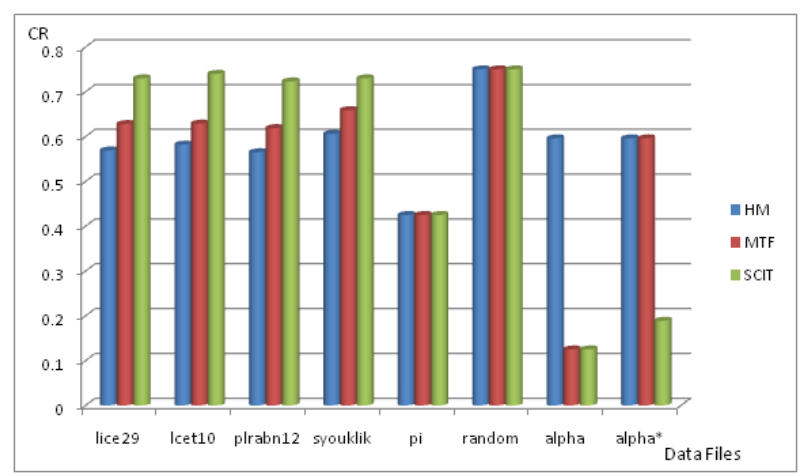

Fig. 3. Performance Comparisons based on Compression Ratio (CR).

alone, and again the SCIT will do better than Huffman alone. But still there are some special alphanumeric character compositions that cannot be affected by BWT technique. For example applying BWT to those files pi.txt, random.txt, and alpha.txt does not give effective change at all. But we can see applying BWT to files syoulik.txt, and alpha*.txt and of course to others will transform them and leads into better compression performance. What we can conclude from these observations is all the methods have their own positive and weak sides. Another critical issue with BWT is that it takes more processing time and needs more main memory for processing.

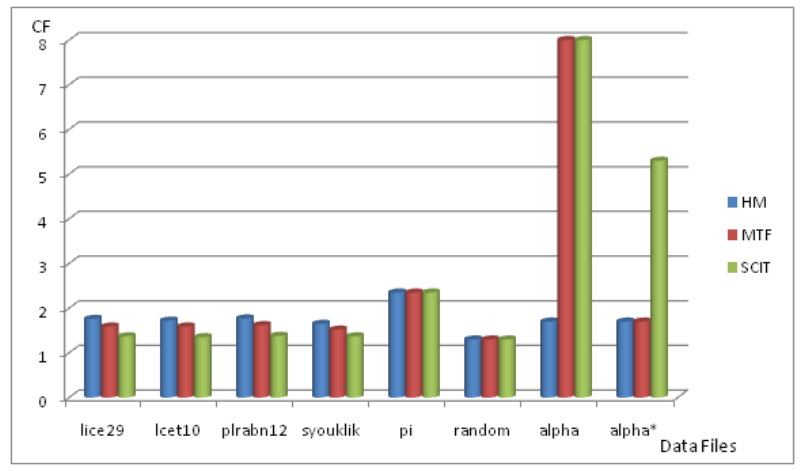

Fig. 4. Performance Comparisons based on Compression Factor $(\mathrm{CF})$.

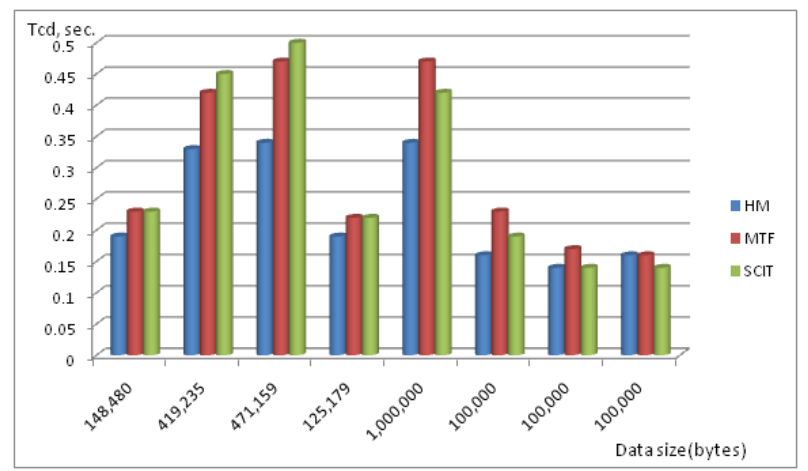

Fig. 5. Performance Comparisons based on Compression and Decompression Time (Ted).

Figure 6, is similar to Figure 2, except it is for multistep compression process using BWT and for the special case file compositions (pi.txt, random.txt, alpha.txt, and alpha*.txt).

\section{CONCLUSIONS}

As data is generated at large volume and data transfer rate is becoming essential in this digital and IOT world, some means of mechanism is required to solve the storage, latency, speed, bandwidth, security and energy problems. Data compression basically aims at minimizing the size of input data so that contribute to solve or min- 


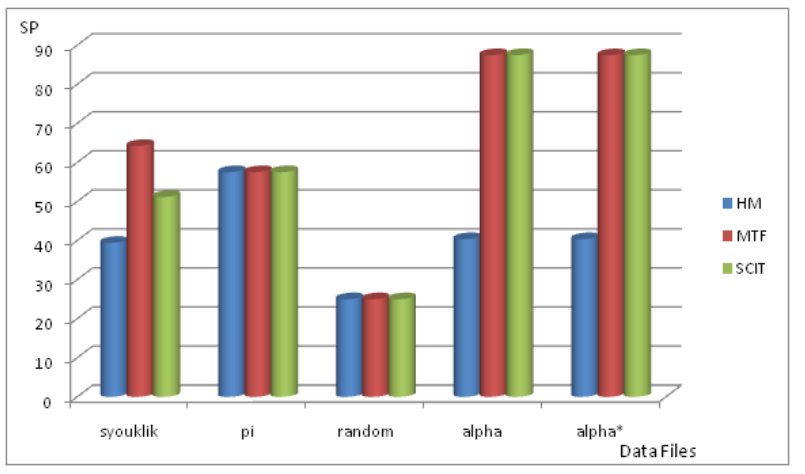

Fig. 6. Performance Comparisons for multistep compression with BWT based on SP.

imize the stated problems in data communication and data storage scenarios. In other words data compression is becoming an integral part of the modern information storage and retrieval systems. Several compression techniques have been developed and implemented for different formats of data files, text, image, video, audio, etc. The research on data compression methods is still continuing as there are gaps to be covered. In this paper, using the proposed SCIT technique, it has been tried to fill some gaps identified in data compression transforming techniques for some special cases at byte/character level. As a conclusion, future works can be carried on an efficient and optimal coding technique (though difficult to avoid the multistep coding techniques approach) for all types of file types (structured, unstructured) so that solve one of the Big Data issues.

\section{REFERENCES}

[1] David Salmon, Data compression, The Complete Reference, 4th Edition.

[2] Stefan Buttcher, Information Retrieval: Implementing and Evaluating Search Engines.

[3] Debra A. Lelewer, Data Compression.

[4] Timothy C. Bell et al, 1990. Text Compression.

[5] Sebastian Deorowicz, Universal lossless data compression algorithms.

[6] David Salomon, Data Compression The Complete Reference 4th edition.

[7] Colt McAnlis \& Aleks Haecky, Understanding Compression, Data Compression for Modern Developers.

[8] Dwi Suarjaya, 2012. A New Algorithm for Data Compression Optimization, JACSA Vol. 3, No.8.

[9] David Salmon et al, Hand Book of Data Compression, 5th Edition.

[10] Rakesh Mohanty, An Improved Move-To-Front (IMTF) Offline Algorithm for the List Accessing Problem.

[11] Khalid Sayood, Introduction to Data Compression, 3rd Edition.

[12] L. Hanzo, R. G. Maunder, 2010. Near-Capacity Variable Length Coding.

[13] Arup Kumar Bhattacharjee, "Comparison Study of Lossless Data Compression Algorithms for Text Data", IOSR Journal of Computer Engineering (IOSR-JCE) Vol.11, May- Jun. 2013, pp 16-19.

[14] Shrusti Porwal, "Data Compression Methodologies for Lossless Data and Comparison between Algorithms", IJESIT, Volume 2, Issue 2, March 2013.

[15] Ritu Antil, "Analysis and Comparison of various lossless Compression Techniques", IJRASET, Vol.2, Issue 3, March 2014. 
Table 3. Compression result using BWT $\rightarrow \mathrm{HM}$, BWT $\rightarrow \mathrm{MTT} \rightarrow \mathrm{HM}$, BWT $\rightarrow \mathrm{SCIT} \rightarrow \mathrm{HM}$

\begin{tabular}{|c|c||c|c|c||c|c|c|c|c|c|}
\hline \multirow{2}{*}{$\begin{array}{c}\text { Input } \\
\text { File }\end{array}$} & \multirow{2}{*}{$\begin{array}{c}\text { Input File } \\
\text { Size(Bytes) }\end{array}$} & \multicolumn{3}{|c|}{ Huffman } & \multicolumn{3}{c|}{ MTF } & \multicolumn{3}{c|}{ SCIT } \\
\hline syoulik & 125,179 & 75,806 & 0.606 & 39.44 & 44,762 & 0.358 & 64.24 & 61,112 & 0.488 & 51.18 \\
\hline & & & & & & & & & & \\
\hline pi & 124,928 & 53,051 & 0.424 & 57.53 & 53,057 & 0.424 & 57.53 & 53,041 & 0.424 & 57.54 \\
\hline random & 100,000 & 75,000 & 0.750 & 25.00 & 75,000 & 0.750 & 25.00 & 75,000 & 0.750 & 25.00 \\
\hline alpha & 100,000 & 59,615 & 0.596 & 40.39 & 12,516 & 0.125 & 87.49 & 12,500 & 0.125 & 87.50 \\
\hline alpha $^{*}$ & 100,000 & 59,615 & 0.596 & 40.39 & 12,525 & 0.125 & 87.47 & 12,500 & 0.125 & 87.48 \\
\hline
\end{tabular}

